\title{
Frequency and Nature of Infectious Risk Moments During Acute Care Based on the INFORM Structured Classification Taxonomy
}

\author{
Lauren Clack, MSc; ${ }^{1}$ Simone Passerini, BScN $;{ }^{1}$ Aline Wolfensberger, MD $;{ }^{1}$ Hugo Sax, MD; ${ }^{1, a}$ Tanja Manser, $\mathrm{PhD}^{2, \mathrm{a}}$
}

\begin{abstract}
ов јестіve. In this study, we sought to establish a comprehensive inventory of infectious risk moments (IRMs), defined as seemingly innocuous yet frequently occurring care manipulations potentially resulting in transfer of pathogens to patients. We also aimed to develop and employ an observational taxonomy to quantify the frequency and nature of IRMs in acute-care settings.
\end{abstract}

DESIGN. Prospective observational study and establishment of observational taxonomy.

SETTING. Intensive care unit, general medical ward, and emergency ward of a university-affiliated hospital.

PARTICIPANTs. Healthcare workers (HCWs).

MEтноDS. Exploratory observations were conducted to identify IRMs, which were coded based on the surfaces involved in the transmission pathway to establish a structured taxonomy. Structured observations were performed using this taxonomy to quantify IRMs in all 3 settings.

RESULts. Following 129.17 hours of exploratory observations, identified IRMs involved HCW hands, gloves, care devices, mobile objects, and HCW clothing and accessories. A structured taxonomy called INFORM (INFectiOus Risk Moment) was established to classify each IRM according to the source, vector, and endpoint of potential pathogen transfer. We observed 1,138 IRMs during 53.77 hours of structured observations (31.25 active care hours) for an average foundation of 42.8 IRMs per active care hour overall, and average densities of 34.9, 36.8, and 56.3 IRMs in the intensive care, medical, and emergency wards, respectively.

CONCLUSIONS. Hands and gloves remain among the most important contributors to the transfer of pathogens within the healthcare setting, but medical devices, mobile objects, invasive devices, and HCW clothing and accessories may also contribute to patient colonization and/or infection. The INFORM observational taxonomy and IRM inventory presented may benefit clinical risk assessment, training and education, and future research.

Infect Control Hosp Epidemiol 2018;39:272-279

Healthcare-associated infections (HAIs) remain a major threat to patient safety. A significant proportion of such infections are likely preventable through the application of infection prevention measures, ${ }^{1-4}$ such as those aiming to reduce the transmission of pathogens that may lead to patient colonization or infection. ${ }^{5}$ Hand hygiene, for example, is widely recognized as one of the most effective practices to reduce infection rates and patient colonization with multidrug-resistant bacteria by reducing the transmission of microorganisms. ${ }^{6}$ Strong evidence also suggests that environmental contamination of surfaces and objects contribute to $\mathrm{HAI},{ }^{7-12}$ yet the behavioral focus of such studies is often limited to hand hygiene and environmental cleaning. While the practice of hand hygiene has been increasingly studied over the last decade for its role in infection prevention, considerably less knowledge exists regarding other important infection-related behaviors.
A growing body of evidence suggests that practices beyond those addressed by hand hygiene may be relevant in the transmission of microorganisms that results in patient colonization and infection, such as handling of mobile objects, ${ }^{13,14}$ healthcare worker (HCW) private $^{15}$ and professional attire, ${ }^{16,17}$ and medical devices. ${ }^{11,14,18}$ Therefore, we hypothesize that an important portion of infectious risks lie in infectious risk moments (IRM), defined as seemingly innocuous, yet frequently occurring care manipulations that potentially result in the transfer of pathogens. Such IRM include yet go beyond existing indications for hand hygiene. ${ }^{13}$

The design of infection prevention strategies that consider a broad range of infectious risks must begin with systematic identification and classification of IRMs. In a 2-part project, we conducted (1) exploratory observations to establish a comprehensive inventory of potential IRMs, which served as

Affiliations: 1. Department of Infectious Diseases and Hospital Epidemiology, University Hospital Zurich, University of Zurich, Zurich, Switzerland; 2. Institute for Patient Safety, University Hospital Bonn, Bonn, Germany.

${ }^{\text {a }}$ Senior authors of equal contribution.

Received August 24, 2017; accepted December 22, 2017

(c) 2018 by The Society for Healthcare Epidemiology of America. This is an Open Access article, distributed under the terms of the Creative Commons Attribution licence (http://creativecommons.org/licenses/by/4.0/), which permits unrestricted re-use, distribution, and reproduction in any medium, provided the original work is properly cited. All rights reserved. 0899-823X/2018/3903-0004. DOI: 10.1017/ice.2017.326 
a basis for developing a taxonomy for structured observations and (2) structured observations to quantify the frequency and nature of IRMs in 3 distinct typical healthcare settings.

\section{METHODS}

\section{Design}

We conducted a prospective observational study in 2 parts. First, we conducted live exploratory observations to identify a wide range of potential IRM and to establish a structured taxonomy called INFORM (INFectiOus Risk Moment) for identifying and classifying IRMs. Second, we conducted live structured observations based on the INFORM taxonomy. Parts of this methodology have been pilot tested previously. ${ }^{13}$ The observations reported in the current manuscript do not include the pilot observations.

\section{Setting}

An intensive care unit (ICU), general medical ward, and emergency ward, including trauma unit, located at a 900-bed, university-affiliated, tertiary-care hospital were purposefully sampled to represent a broad range of care activities and potential infectious risks. All healthcare workers (HCWs) from the participating wards were included in the study. The study hospital has a well-established infection prevention and control (IPC) group with extensive state-of-the-art, written IPC standard operating procedures, weekly IPC rounds, and a designated IPC nurse consultant for each hospital ward.

\section{Exploratory Observations}

Observers with backgrounds in nursing (C.D.A. and V.G.) and human factors/psychology (L.C.) and extensive experience conducting observations for patient safety research carried out exploratory observations in all 3 settings. Field notes documented the care processes observed and any potential IRMs, which were operationally defined as behaviors potentially resulting in the transmission of pathogens that may result in patient colonization or infection. The observers discussed all identified potential IRMs regularly throughout the exploratory observation period together with a senior infection prevention physician (H.S.) and all potential IRM were collected in a database.

Based on the definition of IRMs and following the hand hygiene literature, IRMs were limited to moments resulting in potential transfer of pathogens to patients and their immediate surroundings (eg, bedding), rather than the larger translocation of microorganisms throughout the healthcare environment. For example, an HCW entering a patient room then, without doing hand hygiene, touching the patient's bedside monitor to silence an alarm (a behavior that occurs often and may introduce nonpatient flora to the patient environment) was not considered an IRM. Only behaviors that resulted in potential transfer of pathogens directly to the patient were considered.
We distinguished between noncritical patient sites (eg, intact skin, intact dressings, patient clothing), critical patient sites, defined as "body sites or medical devices that have to be protected against microorganisms potentially leading to $\mathrm{HAI}^{\mathrm{\prime \prime}}$ (eg, mucous membranes, catheter insertion sites, or open wounds), and patient bedding. Exploratory observations were conducted until saturation was achieved in each setting, that is, until no new IRMs were observed.

\section{Structured Observation Taxonomy and Mobile Observation Tool Validation}

Following exploratory observations, all IRMs were extracted from field notes and were systematically coded according to the source, vector, and endpoint from, through, and to which pathogens were transferred, respectively. This structure was used to establish the INFORM classification taxonomy, on which structured observations were based (Figure 1). A mobile observation tool based on the INFORM taxonomy was programmed with Filemaker 14 (FileMaker, Santa Clara, CA). To ensure the quality of observations, 2 observers (L.C. and S. P.) validated the mobile observation tool during a 1-month test period. The percentage of agreement between the 2 observers was calculated to measure sensitivity (detection of the same IRM) and Cohen's $\kappa$ was calculated to determine interobserver agreement (ie, consistent classification of IRM) using STATA version 14 software (StataCorp, College Station, TX).

\section{Structured Observations}

Structured observations were carried out in the same 3 clinical settings using the mobile observation tool. Two observers (L.C. and S.P.) conducted live, structured observations in parallel to ensure systematic documentation of all IRMs. Structured observations targeted periods of active patient care, and both observers focused on the same HCW at once. Observation sessions of 30-60 minutes were deliberately conducted at different times throughout the workday to include many different HCWs who performed a diverse range of care tasks for multiple patients during each session. During live observations, both observers independently noted the source, vector, and endpoint of pathogens for each IRM according to the observational taxonomy as well as demographic information about the HCW being observed (ie, gender and professional category) and contextual information (ie, date, time, ward name, and patient isolation status) using the mobile observation tool (Appendix 1). No identifying patient or HCW data were collected during observations. For each observation period, we recorded the total amount of observation time, as well as the amount of active patient care time to calculate the density of IRMs per setting. Following each structured observation session, all observed IRMs were compared between the 2 observers, and any discrepancies were discussed until a consensus agreement was achieved. Frequent discussion among researchers to achieve consensus after each observation period was maintained throughout the study to ensure quality and to avoid drift between observers. 
Ethics

The Cantonal Ethics Committee of Zurich formally waived the ethics requirement for this study (KEK-StV-Nr.73/14). Participation in observations was voluntary, and HCWs were free to opt out or stop observations at any time without providing justification.

\section{RES ULTS}

\section{Exploratory Observations}

A total of 129.17 hours of exploratory observations resulted in the identification of 292 unique IRMs. Identified IRMs included moments of potential direct contact transmission (potentially infected or colonized HCW to patient) as well as potential indirect contact transmission via vectors such as care devices, mobile objects, and HCW clothing and accessories. Following exploratory observations, IRMs were systematically coded according to the source, vector, and endpoint of potential pathogen transfer, and these codes formed the basis of the INFORM structured taxonomy (Figure 1).
Structured Observation Taxonomy and Mobile Observation Tool Validation

The 3-level taxonomy begins with classification of surfaces (loci) involved in the observed IRM according to source, vector, or endpoint of potential pathogen transfer (level 1: locus), then assigns each source, vector, and endpoint to a main category (level 2: surface), and specifies the exact nature (level 3: surface detail). Each observed IRM is then represented as a transmission chain composed of 3 loci (source, vector, and endpoint), with each locus having 2 levels of detail (surface and surface detail). Table 1 lists examples of archetypal observed and classified IRMs for each of the observed vectors.

During the 1-month test of the taxonomy using the mobile observation tool (5.5 hours of active patient care), observers 1 and 2 detected $123(78.9 \%)$ and $118(75.6 \%)$ of all observed IRMs, respectively. Based on this detection rate, the decision was made to have 2 observers present for all structured observations to ensure the highest possible sensitivity. For moments identified by both observers during the pilot test, the Cohen's $\kappa$ measure of interobserver agreement was 0.75 , indicating substantial agreement between individual observers. ${ }^{20}$

\begin{tabular}{|c|c|}
\hline & Source \\
\hline (Source) & (Source detail ) \\
\hline Environment & $\begin{array}{l}\text { Bedside Table, Curtains, } \\
\text { Floor, Lamp, Outside } \\
\text { Patient Room, Paper } \\
\text { Patient Records, Partition } \\
\text { Walls, Patient Bed, } \\
\text { Trolley, Other }\end{array}$ \\
\hline Gloves & HCW Gloves \\
\hline Hands & HCW Hands \\
\hline $\begin{array}{l}\text { Healthcare } \\
\text { Worker }\end{array}$ & $\begin{array}{l}\text { Body, Clothing, Face, } \\
\text { Hair, Other }\end{array}$ \\
\hline $\begin{array}{l}\text { Invasive } \\
\text { Device }\end{array}$ & $\begin{array}{l}\text { IV Tubes, Mechanical } \\
\text { Ventilator, Suction } \\
\text { Catheter, Other }\end{array}$ \\
\hline $\begin{array}{l}\text { Medical } \\
\text { Device }\end{array}$ & $\begin{array}{l}\text { Bedside Monitor, Blood- } \\
\text { Pressure Cuff, Blood- } \\
\text { Pressure Monitor, ECG, } \\
\text { Infusion Pump, Non- } \\
\text { Invasive Ventilator, } \\
\text { Stethoscope, } \\
\text { Thermometer, } \\
\text { Ultrasound, Ventilator } \\
\text { Monitor, X-Ray, Other }\end{array}$ \\
\hline Mobile Object & $\begin{array}{l}\text { Flashlight, Mobile Phone, } \\
\text { Pen, Secretions, Tape } \\
\text { Dispenser, Toilet Brush, } \\
\text { Washcloth, Other }\end{array}$ \\
\hline Other Patient & $\begin{array}{l}\text { Critical Site, Environment, } \\
\text { Intact Skin }\end{array}$ \\
\hline $\begin{array}{l}\text { Patient } \\
\text { Critical Site }\end{array}$ & $\begin{array}{l}\text { Airways, Bloodstream, } \\
\text { Mucous Membrane Face, } \\
\text { Mucous Membrane } \\
\text { Genitals, Mucous } \\
\text { Membrane Rectum, Open } \\
\text { Wound, Uncapped CVC } \\
\text { Hub, Uncapped IV Line, } \\
\text { Urethra, Other }\end{array}$ \\
\hline $\begin{array}{l}\text { Patient Intact } \\
\text { Skin }\end{array}$ & $\begin{array}{l}\text { Contaminated Skin, } \\
\text { Head, Lower Limbs, } \\
\text { Trunk, Upper Limbs }\end{array}$ \\
\hline $\begin{array}{l}\text { Unknown } \\
\text { Status }\end{array}$ & No Disinfection Observed \\
\hline
\end{tabular}

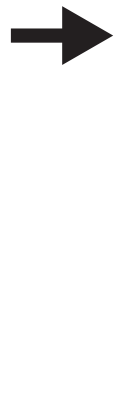

\begin{tabular}{ll}
\hline \multicolumn{1}{c}{ Vector } \\
\hline (Vector) & (Vector detail) \\
\hline Gloves & Don Gloves Without HH, \\
& Non-Sterile Gloves, Remove \\
& Gloves Without HH, Sterile \\
& Gloves \\
\hline Hands & HCW Hands, Patient Hands
\end{tabular}

\begin{tabular}{l} 
(Endpoint) \\
\hline Critical Site
\end{tabular}

Endpoint

Critical Site Airways, Arterial 3-Way Valve, Arterial Insertion Site, Arterial Lumen Port, Bloodstream, CVC Insertion Site, CVC Line 3-Way Valve, CVC Line-Infusion Connection, CVC Lumen Port, Feeding Tube, Mucous Membrane Face, Mucous Membrane Genitals, Mucous Membrane Rectum, Mucous Membrane Urethra, Open Wound, PVC Insertion Site, PVC Line 3-Way Valve, PVC Line-Infusion Connection, PVC Lumen Port, Urinary Catheter, Other

$\begin{array}{ll}\text { Healthcare } & \text { Badge, HCW Private } \\ \text { Worker } & \text { Clothing, HCW White }\end{array}$
g, HCW White $\begin{array}{ll}\text { Clothing, Watch } \\ \text { Invasive } & \text { Arterial Catheter Tip, CVC }\end{array}$ Device Tip, Invasive Ventilator, IV Tubes, Needle/Cannula, PVC Tip, Suction Catheter, Thoracic Tube, Uncapped Hub, Urinary Catheter Tip, Ventilation Filter, Other

Medical Blood-Pressure Cuff, ECG,
Infusion Pump, Non-Invasive Ventilator, Stethoscope, Thermometer, Ultrasound, XRay, Other

$\begin{array}{ll}\text { Device } & \text { Infusion Pump, Non-Invasiv } \\ & \text { Ventilator, Stethoscope, } \\ & \text { Thermometer, Ultrasound, X- } \\ & \text { Ray, Other }\end{array}$
$\begin{array}{ll}\text { Non-Critical } & \text { Head, Lower Limbs, Trunk, Upper } \\ \text { Site } & \text { Limbs, Catheter Dressing, Patient }\end{array}$ $\begin{array}{ll}\text { Son-Critical } & \text { Head, Lower Limbs, Trunk, Uppe } \\ \text { Site } & \text { Limbs, Catheter Dressing, Patien }\end{array}$ \begin{tabular}{ll} 
& Clothing, Wound Dressing, Other \\
\hline Patient Bed & Bedding, Pillow
\end{tabular}

$\begin{array}{ll}\text { Mobile } & \text { Bedding, Dressing or } \\ \text { Object } & \text { Bandage, Flashlight, Medical }\end{array}$

Tape, Mobile Phone, Pen,

Secretions, Tape Dispenser,

Tourniquet, Transfer, Board,

Transfer Cannula, Washcloth, Wristwatch, Other 
TABLE 1. Example Coding of Archetypal Infectious Risk Moments Using the INFORM Structured Taxonomy

Gloves: An HCW wearing gloves removes and discards the dressing from a patient's open wound, his gloves contact the open wound, then, without changing gloves, he touches the insertion site of the same patient's urinary catheter.
Level 1: Locus
Source
Vector
Gloves
Level 2: Surface
Patient critical site
Nonsterile gloves
Endpoint
Critical site
Open wound
Urinary catheter

Healthcare worker: While adjusting the electrocardiography suction nodes to a patient's upper limbs, an HCW leans over the patient and his badge touches the intact skin of the patient's arm.
Level 1: Locus
Source
Vector
Level 2: Surface
Unknown status
Level 3: Surface detail
No disinfection observed
Healthcare worker
Badge
Endpoint
Noncritical site
Upper limbs

Invasive device: An HCW inserts an arterial catheter without having disinfected the skin of the insertion site.

Level 1: Locus

Source

Vector

Endpoint

Level 2: Surface

Patient intact skin

Level 3: Surface detail

Contaminated skin

Invasive device

Arterial catheter tip

Critical site

Bloodstream

Medical device: An HCW carries a stethoscope around her neck and the chest piece comes into contact with her own skin, then, without disinfection, she uses the stethoscope to auscultate the patient.

$\begin{array}{llll}\text { Level 1: Locus } & \text { Source } & \text { Vector } & \text { Endpoint } \\ \text { Level 2: Surface } & \text { Healthcare worker } & \text { Medical device } & \text { Noncritical site } \\ \text { Level 3: Surface detail } & \text { Body } & \text { Stethoscope } & \text { Trunk }\end{array}$

Mobile object: Medical-grade adhesive tape is attached to bedrails prior to being used to secure the gauze of a wound dressing onto the patient's skin.

Level 1: Locus

Level 2: Surface

Source

Environment

Vector

Level 3: Surface detail

Patient bed

Mobile object

Endpoint

Medical tape

Noncritical site

Wound dressing

\section{Structured Observations}

Following validation of the taxonomy using the mobile observation tool, 53.77 hours of structured observations ( 31.25 hours of active care) were conducted, during which 1,338 IRM were identified. The average densities of IRMs per active care hour were 42.8 overall, and $34.9,36.8$, and 56.3 in the intensive care, medical, and emergency wards, respectively. We identified 566 unique IRMs, which fell into 71 main categories according to level 2 of the structured taxonomy. A comprehensive inventory of observed IRMs appears in Table 2.

The vectors in the identified IRMs included hands $(n=596$; $44.54 \%)$, gloves $(n=457 ; 34.16 \%)$, medical devices $(n=115$; $8.59 \%)$, mobile objects $(\mathrm{n}=102 ; 7.62 \%)$, invasive devices $(\mathrm{n}=53 ; 3.96 \%)$, and HCW clothing and accessories $(\mathrm{n}=15$; $1.12 \%$ ). Overall, $25.8 \%$ of IRM concerned moments of potential transmission of pathogens to a critical site, described in detail in Table 2A. Among the 217 IRMs dealing with medical devices and mobile objects as vectors, 143 IRMs (65.90\%) involved the lack of disinfection of a device or object prior to patient contact. The 3 most frequently occurring IRMs per clinical setting are described in detail in Table 3.

\section{I S C U S S I O N}

Hands and gloves continue to be among the most important contributors to the transfer of pathogens in the healthcare setting. Nonetheless, we identified moments dealing with other vectors such as medical devices, mobile objects, invasive devices, and HCW clothing and accessories, which may also contribute to patient colonization and/or infection. While previous studies have shown that indications for hand hygiene occur between 8 per hour in pediatric wards and 30 per hour in ICUs, ${ }^{21,22}$ we found that IRMs occurred with a frequency of 42.8 IRM per active care hour overall and up to 56.3 IRM per active care hour in emergency settings. Similar to opportunities for hand hygiene, the high frequency with which IRMs occur suggests that the cumulative risk of negative patient outcomes due to IRMs may be significant, although the risk of patient infection or colonization with multiresistant pathogens at any single IRM may be low. The fact that $25.8 \%$ of IRMs concerned moments of potential pathogen transfer to critical patient sites further highlights the clinical relevance of IRM for infection prevention.

The structured observations in this study were targeted to moments resulting in potential pathogen transfer to the patient, as opposed to movement of pathogens around the larger healthcare environment. Our exploratory observations nonetheless revealed that pathogen transfer from outside to inside the patient zone likely occurred, for example when coming from one patient to silence an alarm on another patient's monitor without hand hygiene, or when transporting mobile objects that come into contact with multiple consecutive patients during clinical rounds. These findings are 
TABLE 2. Inventory and Observed Frequency of All Infectious Risk Moments per Care Setting by (A) Critical Site and

(B) Noncritical Site

\begin{tabular}{|c|c|c|c|c|c|c|c|c|}
\hline \multicolumn{2}{|l|}{ Source } & \multicolumn{2}{|l|}{ Pathway } & Endpoint & \multicolumn{2}{|c|}{ All ICU } & MED & El \\
\hline \multicolumn{9}{|c|}{$\begin{array}{l}\text { A. Infectious Risk Moments Involving Transfer to Critical Patient } \\
\text { Sites }\end{array}$} \\
\hline Environment & $\rightarrow$ & Gloves & $\rightarrow$ & Critical site & 99 & 36 & 35 & \\
\hline $\begin{array}{l}\text { Medical } \\
\text { device }\end{array}$ & $\rightarrow$ & Gloves & $\rightarrow$ & Critical site & 46 & 28 & 3 & \\
\hline Mobile object & $\rightarrow$ & Gloves & $\rightarrow$ & Critical site & 20 & 14 & 3 & \\
\hline $\begin{array}{l}\text { Patient intact } \\
\text { skin }\end{array}$ & $\rightarrow$ & Gloves & $\rightarrow$ & Critical site & 17 & 8 & 3 & \\
\hline $\begin{array}{l}\text { Healthcare } \\
\text { worker }\end{array}$ & $\rightarrow$ & Gloves & $\rightarrow$ & Critical site & 15 & 11 & 1 & \\
\hline $\begin{array}{l}\text { Invasive } \\
\text { device }\end{array}$ & $\rightarrow$ & Gloves & $\rightarrow$ & Critical site & 1 & 0 & 0 & \\
\hline Other patient & $\rightarrow$ & Gloves & $\rightarrow$ & Critical site & 1 & 1 & 0 & \\
\hline Environment & $\rightarrow$ & Hands & $\rightarrow$ & Critical site & 41 & 17 & 12 & \\
\hline $\begin{array}{l}\text { Medical } \\
\text { device }\end{array}$ & $\rightarrow$ & Hands & $\rightarrow$ & Critical site & 24 & 12 & 3 & \\
\hline $\begin{array}{l}\text { Healthcare } \\
\text { worker }\end{array}$ & $\rightarrow$ & Hands & $\rightarrow$ & Critical site & 5 & 2 & 1 & \\
\hline Mobile object & $\rightarrow$ & Hands & $\rightarrow$ & Critical site & 4 & 1 & 3 & \\
\hline $\begin{array}{l}\text { Patient intact } \\
\text { skin }\end{array}$ & $\rightarrow$ & Hands & $\rightarrow$ & Critical site & 2 & 0 & 0 & \\
\hline $\begin{array}{l}\text { Invasive } \\
\text { device }\end{array}$ & $\rightarrow$ & Hands & $\rightarrow$ & Critical site & 1 & 0 & 1 & \\
\hline Gloves & $\rightarrow$ & $\begin{array}{l}\text { Invasive } \\
\text { device }\end{array}$ & $\rightarrow$ & Critical site & 19 & 14 & 2 & \\
\hline $\begin{array}{l}\text { Patient intact } \\
\text { skin }\end{array}$ & $\rightarrow$ & $\begin{array}{l}\text { Invasive } \\
\text { device }\end{array}$ & $\rightarrow$ & Critical site & 13 & 4 & 1 & \\
\hline Environment & $\rightarrow$ & $\begin{array}{l}\text { Invasive } \\
\text { device }\end{array}$ & $\rightarrow$ & Critical site & 12 & 8 & 1 & \\
\hline $\begin{array}{l}\text { Healthcare } \\
\text { worker }\end{array}$ & $\rightarrow$ & $\begin{array}{l}\text { Invasive } \\
\text { device }\end{array}$ & $\rightarrow$ & Critical site & 4 & 1 & 0 & \\
\hline Hands & $\rightarrow$ & $\begin{array}{l}\text { Invasive } \\
\text { device }\end{array}$ & $\rightarrow$ & Critical site & 3 & 3 & 0 & \\
\hline $\begin{array}{l}\text { Patient } \\
\text { critical site }\end{array}$ & $\rightarrow$ & $\begin{array}{l}\text { Invasive } \\
\text { device }\end{array}$ & $\rightarrow$ & Critical site & 1 & 1 & 0 & \\
\hline Gloves & $\rightarrow$ & $\begin{array}{l}\text { Medical } \\
\text { device }\end{array}$ & $\rightarrow$ & Critical site & 3 & 0 & 1 & \\
\hline Hands & $\rightarrow$ & $\begin{array}{l}\text { Medical } \\
\text { device }\end{array}$ & $\rightarrow$ & Critical site & 1 & 1 & 0 & \\
\hline $\begin{array}{l}\text { Unknown } \\
\text { status }\end{array}$ & $\rightarrow$ & $\begin{array}{l}\text { Medical } \\
\text { device }\end{array}$ & $\rightarrow$ & Critical site & 1 & 0 & 1 & \\
\hline Environment & $\rightarrow$ & $\begin{array}{l}\text { Mobile } \\
\text { object }\end{array}$ & $\rightarrow$ & Critical site & 4 & 1 & 1 & \\
\hline $\begin{array}{l}\text { Patient } \\
\quad \text { critical site }\end{array}$ & $\rightarrow$ & $\begin{array}{l}\text { Mobile } \\
\text { object }\end{array}$ & $\rightarrow$ & Critical site & 4 & 0 & 0 & \\
\hline Gloves & $\rightarrow$ & $\begin{array}{l}\text { Mobile } \\
\text { object }\end{array}$ & & Critical site & 1 & 0 & 1 & \\
\hline Hands & $\rightarrow$ & $\begin{array}{l}\text { Mobile } \\
\text { object }\end{array}$ & & Critical site & 1 & 0 & 0 & \\
\hline $\begin{array}{l}\text { Patient intact } \\
\text { skin }\end{array}$ & $\rightarrow$ & $\begin{array}{l}\text { Mobile } \\
\text { object }\end{array}$ & & Critical site & 1 & 1 & 0 & \\
\hline $\begin{array}{l}\text { Unknown } \\
\text { status }\end{array}$ & $\rightarrow$ & $\begin{array}{l}\text { Mobile } \\
\text { object }\end{array}$ & & Critical site & 1 & 2 & 2 & \\
\hline
\end{tabular}

table 2. Continued

\begin{tabular}{|c|c|c|c|c|c|c|c|c|}
\hline \multicolumn{2}{|l|}{ Source } & \multicolumn{2}{|l|}{ Pathway } & Endpoint & \multicolumn{2}{|c|}{ All ICU } & MED & ER \\
\hline \multicolumn{9}{|c|}{$\begin{array}{l}\text { B. Infectious Risk Moments Involving Transfer to Noncritical } \\
\text { Patient Sites }\end{array}$} \\
\hline Environment & $\rightarrow$ & Gloves & $\rightarrow$ & $\begin{array}{l}\text { Noncritical } \\
\text { site }\end{array}$ & 97 & 26 & 24 & \\
\hline $\begin{array}{l}\text { Medical } \\
\text { device }\end{array}$ & $\rightarrow$ & Gloves & $\rightarrow$ & $\begin{array}{l}\text { Noncritical } \\
\text { site }\end{array}$ & 61 & 9 & 3 & \\
\hline Mobile object & $\rightarrow$ & Gloves & $\rightarrow$ & $\begin{array}{l}\text { Noncritical } \\
\text { site }\end{array}$ & 45 & 8 & 10 & \\
\hline $\begin{array}{l}\text { Patient intact } \\
\text { skin }\end{array}$ & $\rightarrow$ & Gloves & $\rightarrow$ & $\begin{array}{l}\text { Noncritical } \\
\text { site }\end{array}$ & 17 & 7 & 1 & \\
\hline $\begin{array}{l}\text { Healthcare } \\
\text { worker }\end{array}$ & $\rightarrow$ & Gloves & $\rightarrow$ & $\begin{array}{l}\text { Noncritical } \\
\text { site }\end{array}$ & 15 & 4 & 1 & \\
\hline $\begin{array}{l}\text { Patient } \\
\text { critical site }\end{array}$ & $\rightarrow$ & Gloves & $\rightarrow$ & $\begin{array}{l}\text { Noncritical } \\
\text { site }\end{array}$ & 9 & 1 & 4 & \\
\hline $\begin{array}{l}\text { Invasive } \\
\text { device }\end{array}$ & $\rightarrow$ & Gloves & $\rightarrow$ & $\begin{array}{l}\text { Noncritical } \\
\text { site }\end{array}$ & 1 & 1 & 0 & \\
\hline Environment & $\rightarrow$ & Hands & $\rightarrow$ & $\begin{array}{l}\text { Noncritical } \\
\text { site }\end{array}$ & 229 & 34 & 91 & \\
\hline Mobile object & $\rightarrow$ & Hands & $\rightarrow$ & $\begin{array}{l}\text { Noncritical } \\
\text { site }\end{array}$ & 92 & 16 & 33 & \\
\hline $\begin{array}{l}\text { Medical } \\
\text { device }\end{array}$ & $\rightarrow$ & Hands & $\rightarrow$ & $\begin{array}{l}\text { Noncritical } \\
\text { site }\end{array}$ & 77 & 21 & 22 & \\
\hline $\begin{array}{l}\text { Healthcare } \\
\text { worker }\end{array}$ & $\rightarrow$ & Hands & $\rightarrow$ & $\begin{array}{l}\text { Noncritical } \\
\text { site }\end{array}$ & 68 & 9 & 40 & \\
\hline $\begin{array}{l}\text { Patient intact } \\
\text { skin }\end{array}$ & $\rightarrow$ & Hands & $\rightarrow$ & $\begin{array}{l}\text { Noncritical } \\
\text { site }\end{array}$ & 17 & 6 & 4 & \\
\hline Other patient & $\rightarrow$ & Hands & $\rightarrow$ & $\begin{array}{l}\text { Noncritical } \\
\text { site }\end{array}$ & 2 & 0 & 2 & 0 \\
\hline Exterior & $\rightarrow$ & Hands & $\rightarrow$ & $\begin{array}{l}\text { Non- } \\
\text { critical } \\
\text { site }\end{array}$ & 1 & 0 & 1 & \\
\hline $\begin{array}{l}\text { Patient } \\
\quad \text { critical site }\end{array}$ & $\rightarrow$ & Hands & $\rightarrow$ & $\begin{array}{l}\text { Noncritical } \\
\text { site }\end{array}$ & 1 & 1 & 0 & \\
\hline $\begin{array}{l}\text { Unknown } \\
\text { status }\end{array}$ & $\rightarrow$ & Hands & $\rightarrow$ & $\begin{array}{l}\text { Noncritical } \\
\text { site }\end{array}$ & 1 & 0 & 1 & \\
\hline $\begin{array}{l}\text { Unknown } \\
\text { status }\end{array}$ & $\rightarrow$ & HCW & $\rightarrow$ & $\begin{array}{l}\text { Noncritical } \\
\text { site }\end{array}$ & 13 & 5 & 1 & \\
\hline $\begin{array}{l}\text { Patient intact } \\
\text { skin }\end{array}$ & $\rightarrow$ & HCW & $\rightarrow$ & $\begin{array}{l}\text { Noncritical } \\
\text { site }\end{array}$ & 2 & 2 & 0 & \\
\hline $\begin{array}{l}\text { Unknown } \\
\text { status }\end{array}$ & $\rightarrow$ & $\begin{array}{l}\text { Medical } \\
\text { device }\end{array}$ & $\rightarrow$ & $\begin{array}{l}\text { Noncritical } \\
\text { site }\end{array}$ & 81 & 0 & 0 & \\
\hline $\begin{array}{c}\text { Healthcare } \\
\text { worker }\end{array}$ & $\rightarrow$ & $\begin{array}{l}\text { Medical } \\
\text { device }\end{array}$ & $\rightarrow$ & $\begin{array}{l}\text { Noncritical } \\
\text { site }\end{array}$ & 13 & 2 & 0 & \\
\hline Hands & $\rightarrow$ & $\begin{array}{l}\text { Medical } \\
\text { device }\end{array}$ & $\rightarrow$ & $\begin{array}{l}\text { Noncritical } \\
\text { site }\end{array}$ & 3 & 0 & 0 & \\
\hline Gloves & $\rightarrow$ & $\begin{array}{l}\text { Medical } \\
\text { device }\end{array}$ & $\rightarrow$ & $\begin{array}{l}\text { Noncritical } \\
\text { site }\end{array}$ & 1 & 2 & 0 & \\
\hline $\begin{array}{l}\text { Patient intact } \\
\text { skin }\end{array}$ & $\rightarrow$ & $\begin{array}{l}\text { Medical } \\
\text { device }\end{array}$ & $\rightarrow$ & $\begin{array}{l}\text { Noncritical } \\
\text { site }\end{array}$ & 1 & 0 & 1 & \\
\hline $\begin{array}{l}\text { Unknown } \\
\text { status }\end{array}$ & $\rightarrow$ & $\begin{array}{l}\text { Mobile } \\
\text { object }\end{array}$ & $\rightarrow$ & $\begin{array}{l}\text { Noncritical } \\
\text { site }\end{array}$ & 43 & 1 & 0 & \\
\hline Environment & $\rightarrow$ & $\begin{array}{l}\text { Mobile } \\
\text { object }\end{array}$ & $\rightarrow$ & $\begin{array}{l}\text { Noncritical } \\
\text { site }\end{array}$ & 17 & 1 & 2 & \\
\hline $\begin{array}{c}\text { Healthcare } \\
\text { worker }\end{array}$ & $\rightarrow$ & $\begin{array}{l}\text { Mobile } \\
\text { object }\end{array}$ & $\rightarrow$ & $\begin{array}{l}\text { Noncritical } \\
\text { site }\end{array}$ & 6 & 0 & 0 & \\
\hline
\end{tabular}


TABle 2. Continued

\begin{tabular}{|c|c|c|c|c|c|c|c|c|}
\hline Source & & Pathway & & Endpoint & All & $\mathrm{ICU}$ & MED & ER \\
\hline $\begin{array}{l}\text { Patient intact } \\
\text { skin }\end{array}$ & $\rightarrow$ & $\begin{array}{l}\text { Mobile } \\
\text { object }\end{array}$ & $\rightarrow$ & $\begin{array}{l}\text { Noncritical } \\
\text { site }\end{array}$ & 4 & 0 & 0 & \\
\hline Gloves & $\rightarrow$ & $\begin{array}{l}\text { Mobile } \\
\text { object }\end{array}$ & $\rightarrow$ & $\begin{array}{l}\text { Noncritical } \\
\text { site }\end{array}$ & 2 & 1 & 0 & \\
\hline $\begin{array}{l}\text { Medical } \\
\text { device }\end{array}$ & $\rightarrow$ & $\begin{array}{l}\text { Mobile } \\
\text { object }\end{array}$ & $\rightarrow$ & $\begin{array}{l}\text { Noncritical } \\
\text { site }\end{array}$ & 2 & 0 & 6 & \\
\hline $\begin{array}{l}\text { Patient } \\
\text { critical site }\end{array}$ & $\rightarrow$ & $\begin{array}{l}\text { Mobile } \\
\text { object }\end{array}$ & $\rightarrow$ & $\begin{array}{l}\text { Noncritical } \\
\text { site }\end{array}$ & 1 & 4 & 0 & \\
\hline Environment & $\rightarrow$ & Gloves & $\rightarrow$ & Patient bed & 7 & 0 & 0 & \\
\hline $\begin{array}{l}\text { Medical } \\
\text { device }\end{array}$ & $\rightarrow$ & Gloves & $\rightarrow$ & Patient bed & 5 & 0 & 0 & \\
\hline $\begin{array}{l}\text { Healthcare } \\
\text { worker }\end{array}$ & $\rightarrow$ & Gloves & $\rightarrow$ & Patient bed & 1 & 0 & 0 & \\
\hline Environment & $\rightarrow$ & Hands & $\rightarrow$ & Patient bed & 18 & 3 & 8 & \\
\hline $\begin{array}{l}\text { Healthcare } \\
\text { worker }\end{array}$ & $\rightarrow$ & Hands & $\rightarrow$ & Patient bed & 5 & 1 & 2 & \\
\hline $\begin{array}{l}\text { Medical } \\
\text { device }\end{array}$ & $\rightarrow$ & Hands & $\rightarrow$ & Patient bed & 5 & 3 & 0 & 2 \\
\hline Mobile object & $\rightarrow$ & Hands & $\rightarrow$ & Patient bed & 3 & 0 & 2 & \\
\hline Environment & $\rightarrow$ & $\begin{array}{r}\text { Invasive } \\
\text { device }\end{array}$ & $\rightarrow$ & Patient bed & 1 & 1 & 0 & \\
\hline $\begin{array}{l}\text { Unknown } \\
\text { status }\end{array}$ & $\rightarrow$ & $\begin{array}{l}\text { Medical } \\
\text { device }\end{array}$ & $\rightarrow$ & Patient bed & 7 & 9 & 26 & 42 \\
\hline $\begin{array}{l}\text { Healthcare } \\
\text { worker }\end{array}$ & $\rightarrow$ & $\begin{array}{l}\text { Medical } \\
\text { device }\end{array}$ & $\rightarrow$ & Patient bed & 1 & 2 & 1 & \\
\hline $\begin{array}{l}\text { Unknown } \\
\text { status }\end{array}$ & $\rightarrow$ & $\begin{array}{l}\text { Mobile } \\
\text { object }\end{array}$ & $\rightarrow$ & Patient bed & 10 & 6 & 12 & 18 \\
\hline Environment & $\rightarrow$ & $\begin{array}{l}\text { Mobile } \\
\text { object }\end{array}$ & $\rightarrow$ & Patient bed & 5 & 5 & 5 & 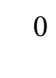 \\
\hline
\end{tabular}

NOTE. ICU, intensive care unit; MED, general medical ward; ER, emergency ward; HCW, healthcare worker.

consistent with other studies demonstrating that HCW hand hygiene compliance prior to initial contact with the patient or the patient environment is suboptimal. ${ }^{23}$ Our results also challenge the "patient zone" concept, which defines the patient and his/her immediate surroundings (eg, bed rails, bedside table, and medical equipment) and frequently touched surfaces (eg, monitors, knobs, and buttons) as the patient zone and assumes that surfaces within the patient zone are colonized by patient flora. ${ }^{19}$ When disinfection is omitted prior to contact with the patient or patient environment, ${ }^{23}$ it is likely that pathogens from the healthcare environment are introduced to these surfaces. Such ambiguity is a major challenge to safe behavior. ${ }^{24}$ For this reason, during observations, we considered that environmental surfaces could potentially harbor pathogenic bacteria regardless of their location inside or outside of the patient zone.

Similarly, our findings are consistent with multiple systematic reviews demonstrating that the frequent movement of healthcare equipment ${ }^{25}$ and care items ${ }^{14}$ between patients, together with suboptimal or missing disinfection of such items, result in the transfer of pathogens between patients. Potential contamination or missing disinfection of medical devices and mobile objects (classified as source = "unknown status" and source detail = "no disinfection observed") accounted for $16.2 \%$ of IRMs observed in this study (Table 2).

The transmission-based observational approach employed in this study, which sought to identify all behaviors potentially resulting in transmission pathogen, differs from traditional rule-based observations that measure compliance with existing local or national guidelines. Observations using the INFORM taxonomy could hence be employed in additional settings, regardless of local guidelines, to identify the most frequently occurring IRMs and to establish local infection prevention priorities.

This study has several limitations. It is possible that being observed influenced HCW behavior during this study. ${ }^{26}$ It is unlikely, however, that this resulted in systematic bias because HCWs were not aware of exactly what was being observed. Observations were limited to contact transmission (ie, the most common mode of transmission ${ }^{5}$ ) and did not consider airborne and droplet transmission. Furthermore, our observations did not consider other behaviors that may also impact infectious risks, such as those interfering with the patient's defense system against infectious risks (eg, immune status, skin integrity, cough reflex, etc) because the associated HCW behavior rarely occurs at the bedside. Moreover, these observations were conducted in a single university hospital located in a high-income setting, which limits the generalizability of our findings. Further exploration of the nature and frequency of IRMs using the INFORM structured observational taxonomy is warranted to assess local priorities for infection prevention efforts in additional care settings. Finally, the risk of transmission during each type of IRM remains unknown. We aimed to bridge this gap through a modified Delphi survey with an international panel of experts in infectious diseases, infection prevention and control, and microbiology, in which experts rated the likelihood of infectious outcomes (eg, colonization, infection) following archetypical IRM. ${ }^{27}$

Despite these limitations, the combination of methods employed in this study was well suited to identify a wide range of potential IRMs and to systematically observe their frequency and nature in multiple healthcare settings. The resulting mobile observation tool featuring the INFORM taxonomy of source, vector, and endpoint of pathogens was useful for the systematic documentation and categorization of IRMs. Further observations based on the INFORM taxonomy may prove useful in other settings to identify the most frequently occurring IRMs, to establish educational content, and to prioritize targeted infection prevention strategies.

\section{ACKNOWLEDGMENTS}

We would like to wholeheartedly thank all healthcare workers for their participation in this study. We also gratefully acknowledge Claudia Dell Apollonia and Verena Gabler for their contributions to data collection during exploratory observations. We also thank Jasmina Bogdanovic and Sabine Greschek for their critical feedback on an advanced draft of this paper. 
TABLE 3. Three Most Frequently Occurring Infectious Risk Moments (IRM) per Clinical Setting

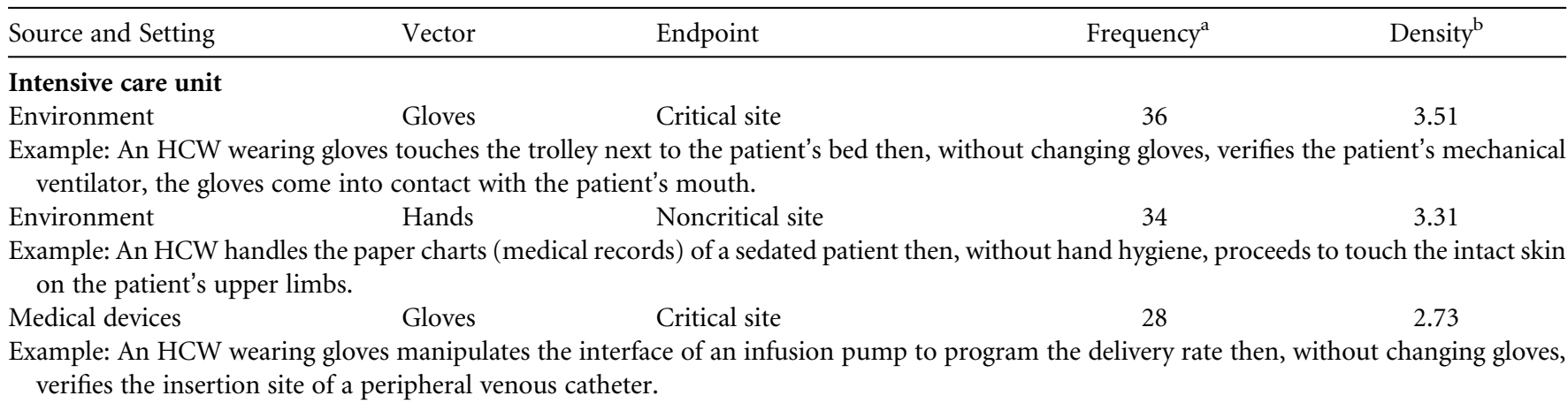

\section{Medical ward}

Environment

Hands Noncritical site

91

8.78

Example: After touching the environment outside of the patient's room, an HCW enters a patient's room and, without doing hand hygiene, shakes the patient's hand.
Healthcare worker
Hands
Noncritical site
40
3.86

Example: An HCW stands with arms crossed, his hands come into contact with his white professional clothing then, without performing hand hygiene, proceeds to examine the patient, touching intact skin on the patient's stomach.

Environment Gloves Critical site

35

3.38

Example: While changing a wound dressing, an HCW wearing gloves touches the surface and drawers of the trolley containing dressing materials, then with the same gloves make contact with the patient's open wound.

\section{Emergency ward}

Environment

Hands Noncritical site

104

9.7

Example: After touching the environment outside of the patient's room, an HCW enters a patient's room and, without performing hand hygiene, shakes the patient's hand.
Medical devices
Gloves
Noncritical site
49
4.62

Example: An HCW wearing gloves touches the electronic interface of an electrocardiography machine (ECG), whose disinfection had not been observed prior to using, then with the same gloves touches the patient's intact skin while applying the ECG nodes to the patient.
Environment
Gloves
Noncritical site
47
4.43

Example: An HCW wearing gloves pulls closed the curtains that divide patient rooms, then, wearing the same gloves, touches the patient's upper limbs.

NOTE. This table presents the 3 most frequently occurring main categories of infectious risk moments (IRMs) based on level 2 of the structured taxonomy.

${ }^{a}$ Number of times the IRM was observed in the indicated setting.

${ }^{\mathrm{b}}$ Frequency per hour of active patient care in the indicated setting.

Financial support: This study was funded by the Swiss National Science Foundation (grant no. 32003B_149474).

Potential conflicts of interest: All authors report no conflicts of interest relevant to this article.

Address correspondence to Lauren Clack, University Hospital Zurich. Rämistrasse 100, HAL 14, B4, 8091 Zurich, Switzerland (Lauren.clack@usz.ch).

\section{SUPPLEMENTARY MATERIAL}

To view supplementary material for this article, please visit https://doi.org/10.1017/ice.2017.326

\section{REFERENCES}

1. Harbarth S, Sax H, Gastmeier P. The preventable proportion of nosocomial infections: an overview of published reports. J Hosp Infect 2003;54:258-266; quiz 321.
2. Umscheid CA, Mitchell MD, Doshi JA, Agarwal R, Williams K, Brennan PJ. Estimating the proportion of healthcare-associated infections that are reasonably preventable and the related mortality and costs. Infect Control Hosp Epidemiol 2011;32:101-114.

3. Zingg W, Holmes A, Dettenkofer M, et al. Hospital organisation, management, and structure for prevention of health-careassociated infection: a systematic review and expert consensus. Lancet Infect Dis 2015;15:212-224.

4. Storr J, Twyman A, Zingg W, et al. Core components for effective infection prevention and control programmes: new WHO evidence-based recommendations. Antimicrob Resist Infect Control 2017;6:6.

5. Siegel JD, Rhinehart E, Jackson M, Chiarello L. Health Care Infection Control Practices Advisory Committee (HICPA). 2007 Guideline for isolation precautions: preventing transmission of infectious agents in healthcare settings. Am J Infect Control 2007; 35(10 Suppl 2):S65-S164. 
6. Allegranzi B, Pittet D. Role of hand hygiene in healthcareassociated infection prevention. J Hosp Infect 2009;73: 305-315.

7. Boyce JM. Environmental contamination makes an important contribution to hospital infection. J Hosp Infect 2007; 65(Suppl 2):50-54.

8. Weber DJ, Anderson D, Rutala WA. The role of the surface environment in healthcare-associated infections. Curr Opin Infect Dis 2013;26:338-344.

9. Duckro AN, Blom DW, Lyle EA, Weinstein RA, Hayden MK. Transfer of vancomycin-resistant enterococci via health care worker hands. Arch Intern Med 2005;165:302-307.

10. Bonten MJ, Hayden MK, Nathan C, et al. Epidemiology of colonisation of patients and environment with vancomycinresistant enterococci. Lancet 1996;348:1615-1619.

11. Schultsz C, Meester HH, Kranenburg AM, et al. Ultra-sonic nebulizers as a potential source of methicillin-resistant Staphylococcus aureus causing an outbreak in a university tertiary care hospital. J Hosp Infect 2003;55:269-275.

12. Fawley WN, Parnell P, Verity P, Freeman J, Wilcox MH. Molecular epidemiology of endemic Clostridium difficile infection and the significance of subtypes of the United Kingdom epidemic strain (PCR ribotype 1). J Clin Microbiol 2005;43:2685-2696.

13. Clack L, Schmutz J, Manser T, Sax H. Infectious risk moments: a novel, human factors-informed approach to infection prevention. Infect Control Hosp Epidemiol 2014;35:1051-1055.

14. Livshiz-Riven I, Borer A, Nativ R, Eskira S, Larson E. Relationship between shared patient care items and healthcare-associated infections: a systematic review. Int J Nurs Stud 2015;52:380-392.

15. Lopez PJ, Ron O, Parthasarathy P, Soothill J, Spitz L. Bacterial counts from hospital doctors' ties are higher than those from shirts. Am J Infect Control 2009;37:79-80.

16. Wiener-Well Y, Galuty M, Rudensky B, Schlesinger $\mathrm{Y}$, Attias D, Yinnon AM. Nursing and physician attire as possible source of nosocomial infections. Am J Infect Control 2011;39:555-559.
17. Treakle AM, Thom KA, Furuno JP, Strauss SM, Harris AD, Perencevich EN. Bacterial contamination of health care workers' white coats. Am J Infect Control 2009;37:101-105.

18. Birnbach DJ, Rosen LF, Fitzpatrick M, Carling P, Munoz-Price LS. The use of a novel technology to study dynamics of pathogen transmission in the operating room. Anesth Analg 2015;120: 844-847.

19. Sax H, Allegranzi B, Uckay I, Larson E, Boyce J, Pittet D. 'My five moments for hand hygiene': a user-centred design approach to understand, train, monitor and report hand hygiene. J Hosp Infect 2007;67:9-21.

20. Landis JR, Koch GG. The Measurement of Observer Agreement for Categorical Data. Biometrics 1977;33:159-117.

21. Pittet D, Mourouga P, Perneger TV. Compliance with Handwashing in a Teaching Hospital. Ann Intern Med 1999;130: 126-130.

22. Hugonnet S, Perneger TV, Pittet D. Alcohol-based handrub improves compliance with hand hygiene in intensive care units. Arch Intern Med 2002;162:1037-1043.

23. Erasmus V, Daha TJ, Brug H, et al. Systematic review of studies on compliance with hand hygiene guidelines in hospital care. Infect Control Hosp Epidemiol 2010;31:283-294.

24. Sax H, Clack L. Mental models: a basic concept for human factors design in infection prevention. J Hosp Infect 2015;89: 335-339.

25. Schabrun S, Chipchase L. Healthcare equipment as a source of nosocomial infection: a systematic review. J Hosp Infect 2006;63: 239-245.

26. Parsons HM. What happened at Hawthorne? New evidence suggests the Hawthorne effect resulted from operant reinforcement contingencies. Science 1974;183:922-932.

27. Clack L, Passerini S, Manser T, Sax H. Likelihood of infectious outcomes following infectious risk moments during patient care-an international expert consensus study and quantitative risk index. Infect Control Hosp Epidemiol 2018;39:280-289. 